\title{
Ion-implanted lanthanum fluoride waveguides
}

\author{
X. Huang ${ }^{\text {a, * }}$, P.J. Chandler ${ }^{a}$, P.D. Townsend ${ }^{a}$, H. Rutt ${ }^{b}$ \\ a Department of Physics, University of Sussex, Falmer, Brighton BN1 9QH, UK \\ ${ }^{b}$ Department of Physics, University of Southampton, Highfield, Southampton SO9 5NH, UK
}

\begin{abstract}
The effects of $\mathrm{He}^{+}$and $\mathrm{H}^{+}$implantation into $\mathrm{LaF}_{3}$ substrates have been investigated at both room temperature and $77 \mathrm{~K}$. At room temperature the surface of the samples becomes black, possibly due to chemical reduction effects, but at low temperature the crystals remain clear and a refractive index change is observed which produces a surface waveguide. The dark mode spectra of these guides have been recorded using the prism coupling technique, and their refractive index profiles have been deduced from the spacing of these modes. The ion implantation produces a thin optical barrier layer of low refractive index at the end of the ion range. This paper discusses the use of multiple-energy implantation to produce a broad optical barrier in order to reduce the tunnelling losses of the guides, and subsequent thermal annealing to reduce absorption and scattering losses caused by colour centre formation in the electronic stopping surface region of the guides. It is concluded that either single- or multiple-energy $\mathrm{He}^{+}$implants can be ideally used to form waveguides, for applications in the blue/UV or red/IR wavelength regions, respectively, with losses of a few $\mathrm{dB} / \mathrm{cm}$.
\end{abstract}

\section{Introduction}

The dielectric crystal Lanthanum Fluoride $\left(\mathrm{LaF}_{3}\right)$ is a laser host material, which can be doped with different rare-earth ions to produce a variety of lasing emissions. For example, Neodymium doped $\mathrm{LaF}_{3}$ may generate infrared down-conversion or ultraviolet and green-red upconversion $[1,2]$, and the first solid state vacuum ultraviolet laser operating at $172 \mathrm{~nm}$ has been constructed in $\mathrm{Nd}^{3+}: \mathrm{LaF}_{3}[3,4]$. For lasing to occur, the gain in the optically pumped medium must exceed the cavity losses. Confinement of the pump and laser modes to the gain region is essential for efficient operation, and so waveguide confinement is potentially superior to a bulk laser system, which has much larger dimensions and mode-sizes. In this paper, we report the fabrication of optical waveguides in $\mathrm{LaF}_{3}$ by ion implantation, and their optimisation for applications at either short or long wavelengths.

\section{Experimental}

Surface and edge polished $\mathrm{LaF}_{3}$ samples have been implanted using a Van der Graaf accelerator with $\mathrm{He}^{+}$and $\mathrm{H}^{+}$ions at energies in the regions of 2.0 and $0.7 \mathrm{MeV}$, respectively. The implant doses were varied from $1 \times 10^{16}$

\footnotetext{
* Corresponding author. Fax: +44 1273 678193; e-mail: x.huang@sussex.ac.uk
}

to $12 \times 10^{16}$ ions $/ \mathrm{cm}^{2}$ and performed at room temperature or liquid nitrogen temperature. The beam was scanned to give a uniform implantation, with an average current density of about $0.5 \mu \mathrm{A} / \mathrm{cm}^{2}$. Post-implant furnace annealing treatments were carried out in 30 minute stages at temperatures up to $380^{\circ} \mathrm{C}$.

Complete dark mode spectra of the implanted samples were measured at $488 \mathrm{~nm}$ and $633 \mathrm{~nm}$ using a standard single coupling prism technique with an expanded laser beam. The mode data were analysed by means of a reflectivity-based calculation and fitted by computer to give analytic refractive index profiles using a parameter optimisation routine [5].

After each implant and thermal annealing treatment, waveguide insertion losses were determined at $488 \mathrm{~nm}$ by means of end coupling with $\times 20$ microscope objective lenses. The waveguide losses were subsequently recorded throughout the visible wavelength region using white light end-coupling, and detection with a Bentham $25 \mathrm{~cm}$ monochromator and photomultiplier system. These loss spectra were calibrated by using the above insertion loss values at $488 \mathrm{~nm}$.

\section{Results and discussion}

After room temperature implantation, either with $\mathrm{He}^{+}$ ions or with $\mathrm{H}^{+}$ions, the surface of the samples became black, possibly due to chemical reduction effects (e.g. loss of fluorine), whereas implants performed at liquid nitrogen 
temperature $(77 \mathrm{~K})$ did not have this problem. Subsequent implants were therefore performed at low temperature.

\subsection{Refractive index profiles}

Fig. 1 shows the refractive index profiles of the implanted crystal surface of a $\mathrm{LaF}_{3}$ sample after a $2 \mathrm{MeV}$ $\mathrm{He}^{+}$implant at $77 \mathrm{~K}$ with dose $3 \times 10^{16}$ ions $/ \mathrm{cm}^{2}$. Optical waveguides can be formed by increasing the refractive index of the guiding region, and/or reducing the refractive index (making an optical barrier) at the boundary of that region. The advantage of the index increased guides is that the optical modes are fully confined, whereas the optical barrier guides may exhibit tunnelling losses, i.e. the optical modes may leak out into the substrate. However, if the barrier is wide or high enough, this loss may not be significant, especially for short wavelength. The index profiles (Fig. 1) show that the implant produces a reduced refractive index barrier, which is about $4.5 \mu \mathrm{m}$ beneath the surface near the end of the ion range. The

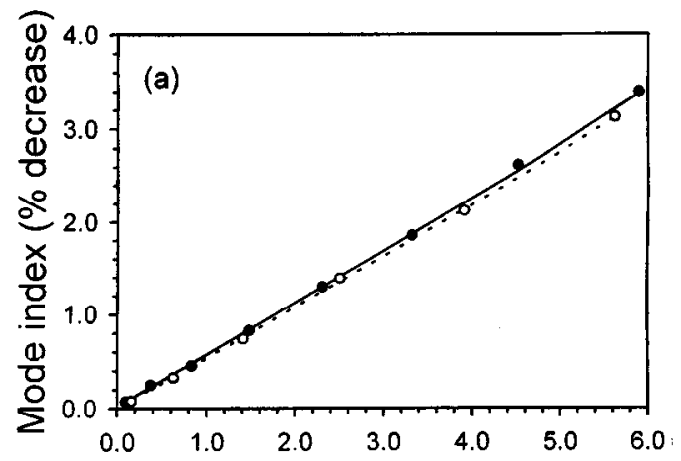

Normalized mode number squared

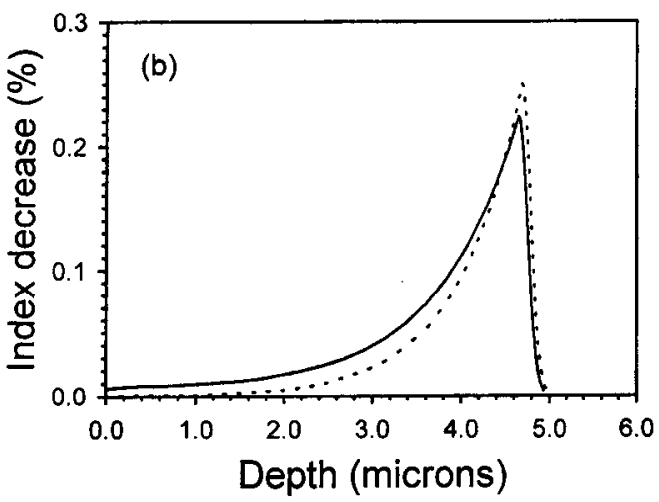

Fig. 1. Refractive index profiles for an implantation using $3 \times 10^{16}$ ions $/ \mathrm{cm}^{2}$ of $2.0 \mathrm{MeV} \mathrm{He}{ }^{+}$at $77 \mathrm{~K}$, measured at $488 \mathrm{~nm}$ (solid) and $633 \mathrm{~nm}$ (dashes). (a) Mode curves: the data points represent the experimental dark mode positions and the curves give the theoretical mode positions corresponding to the fitted refractive index profiles shown in (b). An exponential is chosen as the most suitable function to match the profile.

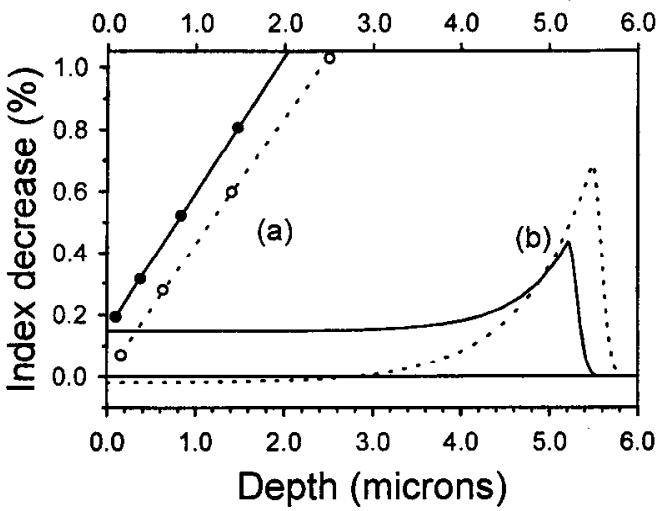

Fig. 2. As for Fig. 1, but using $4 \times 10^{16}$ ions $/ \mathrm{cm}^{2}$ of $0.7 \mathrm{MeV}$ $\mathrm{H}^{+}$at $77 \mathrm{~K}$. There is an obvious dispersive effect between the red and blue indices apparent in the implanted region.

refractive index is almost unchanged near the crystal surface (i.e. the electronic energy transfer region). The barrier is best fitted by an exponential with a height of about $0.2 \%$ index change, and thickness around $0.7 \mu \mathrm{m}$ at half maximum height. Such an index change is small, when compared with the changes in many other ion implanted waveguides. This result suggests that $\mathrm{He}^{+}$implantation may form optical barrier guides in $\mathrm{LaF}_{3}$, but that a tunnelling problem may possibly exist if the barrier can not be improved in height or width.

In order to produce a comparable depth waveguide using hydrogen ions, $0.7 \mathrm{MeV} \mathrm{H}^{+}$was implanted at $77 \mathrm{~K}$ with a dose of $4 \times 10^{16}$ ions $/ \mathrm{cm}^{2}$. The refractive index profiles measured at $633 \mathrm{~nm}$ and $488 \mathrm{~nm}$ are shown in Fig. 2. The one measured at $633 \mathrm{~nm}$ is very similar to those of the $\mathrm{He}^{+}$implanted ones, forming a narrow reduced index barrier at the end of the ion range, which is possibly higher than the $\mathrm{He}^{+}$one. However, the profile measured at 488 nm shows a rather poor confinement, with the added effect of a large index decrease in the guiding region reducing the height of the barrier. One explanation for this change of optical dispersion observed with hydrogen implants is that it is caused by chemical bonding effects. Furthermore, colour centre and defect formation in halides is known to be sensitive to the rate of energy deposition, and so a difference in refractive index effects between $\mathrm{He}^{+}$and $\mathrm{H}^{+}$ beams is understandable for these reasons also. Further implants using $\mathrm{H}^{+}$up to doses of $12 \times 10^{16}$ ions $/ \mathrm{cm}^{2}$ were not able to improve the optical barrier to a significant degree. These results suggest that for the best refractive index confinement, $\mathrm{He}^{+}$implantation is preferable to $\mathrm{H}^{+}$ implantation for making optical waveguides in $\mathrm{LaF}_{3}$.

\subsection{Insertion losses of single-barrier waveguides}

Loss measurements were made on an optical waveguide formed by $\mathrm{He}^{+}$implantation and post-implant ther- 


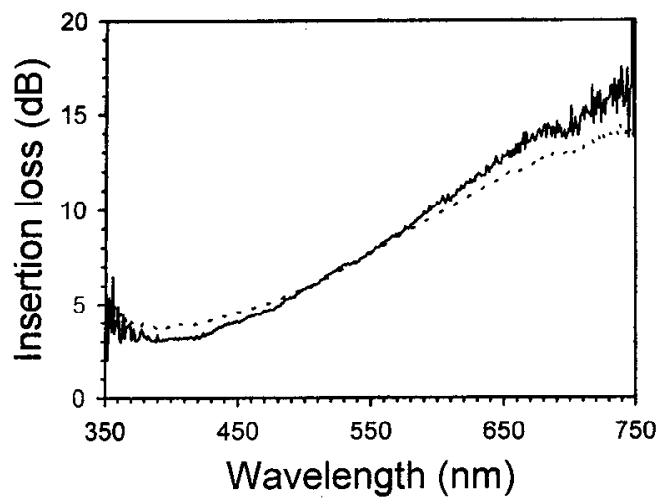

Fig. 3. Insertion loss spectra for a $3 \mathrm{~mm}$ long sample implanted as in Fig. 1, measured with white light and calibrated with a $488 \mathrm{~nm}$ Argon laser. The sample was annealed at (a) $350^{\circ} \mathrm{C}$, dots; (b) $380^{\circ} \mathrm{C}$, solid.

mal annealing up to $380^{\circ} \mathrm{C}$. The absolute end-coupling insertion loss at $488 \mathrm{~nm}$ was determined using an Argon laser, and also the complete visible loss spectra using white light. The spectrum (Fig. 3) shows that the annealed guide has a moderately low loss $(\sim 3 \mathrm{~dB})$ in the blue region, but that the loss increases very significantly with wavelength, up to $\sim 12 \mathrm{~dB}$ at $750 \mathrm{~nm}$. This loss includes the effect of tunnelling through the narrow optical barrier, which increases drastically with wavelength. After a guide has been annealed at a suitable temperature for sufficient time, because the colour centres and defects are removed from the guiding region, the absorption and scattering losses, which have a bigger effect at shorter wavelengths, decrease significantly. However, if the temperature is too high, the tunnelling loss may increase, especially at longer wavelength due to the partial annealing out of the optical barrier. Comparing spectra (a) and (b) of Fig. $3,350^{\circ} \mathrm{C}$ is seen to be about the temperature limit of the annealing, since after $380^{\circ} \mathrm{C}$ annealing, although the colour centre absorption improves further, the tunnelling losses begin to increase. Such a leaky guide can be used in short wavelength applications, but can not be applied in the red/infrared region. An effort was therefore made to increase the index change of the barrier by increasing the implant dose. However, using doses above $3 \times 10^{16}$ ions $/ \mathrm{cm}^{2}$, it was found that after thermal annealing the surface was ruptured by the appearance of small gas bubbles, caused by the diffusion of helium.

\subsection{Insertion losses of $\mathrm{He}^{+}$implanted multiple-barrier waveguides}

An alternative approach to improving the confinement of the optical barrier is to increase its width rather than its height by using multiple implants with slightly different energies. In this case, the multiple barriers produced by the implants are performed as one "wide" barrier to increase

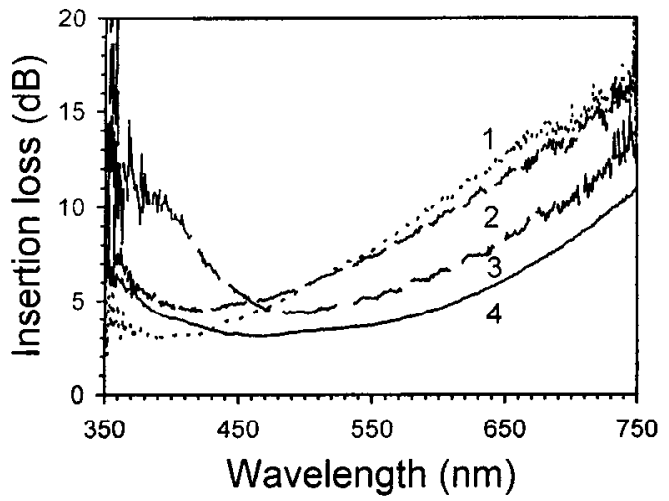

Fig. 4. Insertion loss spectra for a $3 \mathrm{~mm}$ long multiple $\mathrm{He}^{+}$ implanted sample using $2.1 \mathrm{MeV}$ dose $3 \times 10^{16}$ ions $/ \mathrm{cm}^{2}$ plus 2.4, 2.6, $2.8 \mathrm{MeV}$ each dose $1 \times 10^{16}$ ions $/ \mathrm{cm}^{2}$. (1) $2.1 \mathrm{MeV}$, $380^{\circ} \mathrm{C}$ anneal, dots; (2) plus $2.4 \mathrm{McV}, 250^{\circ} \mathrm{C}$ anneal, short dashes; (3) plus $2.6 \mathrm{MeV}, 250^{\circ} \mathrm{C}$ anneal, long dashes; (4) plus $2.8 \mathrm{MeV}$, $300^{\circ} \mathrm{C}$ anneal, solid.

the optical confinement. A bonus from this method is that since the implanted ions are more spread out, the surface lift off problem due to helium diffusion and bubbling is significantly reduced.

Fig. 4 shows the loss spectra in the visible wavelength region of a multiple-implanted sample. It was initially fabricated using $2.1 \mathrm{MeV} \mathrm{He}^{+}$with dose $3 \times 10^{16}$ ions $/ \mathrm{cm}^{2}$ to form the main optical barrier confinement. Subsequently, three more implants were performed, each with a dose of $1 \times 10^{16}$ ions $/ \mathrm{cm}^{2}$ at $2.4,2.6$ and 2.8 $\mathrm{MeV}$, respectively. After each implant, a medium temperature thermal annealing $\left(250^{\circ} \mathrm{C}\right)$ was carried out in order to remove some of the colour centre defects, but retain the guide barrier. The end-coupling loss spectra were measured after each stage in order to see the effect of the barrier broadening. It can be seen from Fig. 4 that the

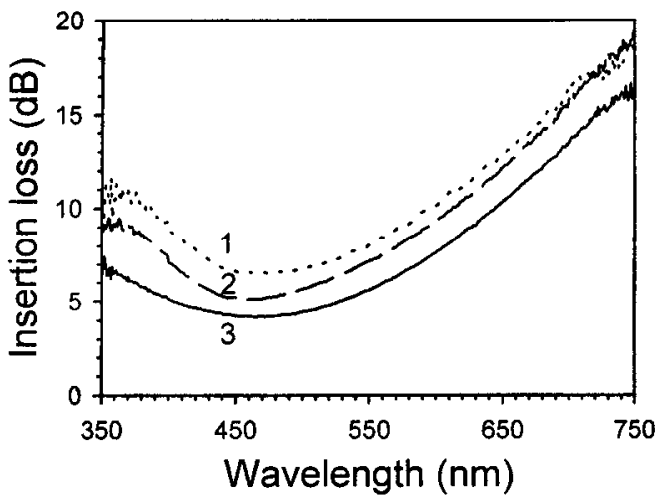

Fig. 5. Insertion loss spectra for a $3 \mathrm{~mm}$ long multiple $\mathrm{He}^{+}$ implanted sample using $2.7,2.5,2.3 \mathrm{MeV}$ each dose $2 \times 10^{16}$ ions $/ \mathrm{cm}^{2}$. All spectra are taken after the final implant, and annealed at: (1) $250^{\circ} \mathrm{C}$, dots; (2) $300^{\circ} \mathrm{C}$, dashes; (3) $350^{\circ} \mathrm{C}$, solid. 
insertion losses in the red/infrared region due to tunnelling have been significantly reduced by a factor of 2 in $\mathrm{dB}$ after four implants and a final annealing at $300^{\circ} \mathrm{C}$. The loss in the blue region due to increased colour centres does not become a significant problem at the doses used.

A second sample was implanted with multiple energy $\mathrm{He}^{+}$to give a broad barrier. The total dose was the same as that in Fig. $4\left(6 \times 10^{16}\right.$ ions $\left./ \mathrm{cm}^{2}\right)$, but it consisted of three equal doses at energies $2.7,2.5$, and $2.3 \mathrm{MeV}$. The guide was annealed in stages from $250^{\circ} \mathrm{C}$ to $350^{\circ} \mathrm{C}$, and insertion loss spectra measured. Fig. 5 shows that the performance of this barrier was not as good as the previous one. More data are needed on the performance of different energy/dose combinations, in order to optimise the confinement of multiple-energy barriers. Overall the data suggest that different implant strategies are needed for UV or IR waveguides in $\mathrm{LaF}_{3}$.

\section{Conclusion}

By means of $\mathrm{He}^{+}$implantation, it is possible to form optical waveguides with a refractive index barrier confinement in $\mathrm{LaF}_{3}$. The guide can be fabricated either with a single barrier for applications in the UV/blue wavelength region, or with multiple-barriers for usage in the red/infrared wavelength region. Further work is needed to determine the ideal parameters of the multiple-energy implants.

Hydrogen implants produce problems which give poor optical confinement, such that insertion losses are too high to measure. However, from the index profile measurements, it may be possible that further work will show their suitability at longer wavelengths.

\section{Acknowledgements}

The financial support of the ORC at Southampton is gratefully acknowledged.

\section{References}

[1] D.K. Fork, F. Armani-Leplingard, M. Lui and R.A. McFarlane, J. Lightwave Technol. 14 (1996) 611-615.

[2] Valerii V. Ter-Mikirtychev and Taiju Tsuboi, Phys. Rev. B Condensed Matter 52 (1995) 15027-15030.

[3] W. Waynant and P.H. Klein, Appl. Phys. Lett. 46 (1985) 14.

[4] W. Waynant, Appl. Phys. B 28 (1982) 205.

[5] P.J. Chandler and F.L. Lama, Opt. Acta 33 (1986) 127-143. 